\title{
Influence of Imposed Optic Flow Characteristics and Intention on Postural Responses
}

\author{
Ana M. F. Barela, José A. Barela, Natália M. Rinaldi, \\ and Diana R. de Toledo
}

\begin{abstract}
This study examined the influence of both optic flow characteristics and intention on postural control responses. Two groups of 10 adults each were exposed to the room's movement either at $0.6 \mathrm{~cm} / \mathrm{s}$ (low velocity group) or $1.0 \mathrm{~cm} / \mathrm{s}$ (high velocity group). All the participants stood in the upright stance inside of a moving room and were informed about the room movement only after the fourth trial as they were asked to resist to its influence. Results revealed that participants from both groups were influenced by the imposed visual stimulus in the first trials, but the coupling strength was weaker for the high velocity group. The request to resist the visual influences decreased visual influences on body sway, but only for the low velocity group. These results indicate that intention might play a role in stimulus influences on body sway but it is stimulus dependent.
\end{abstract}

Keywords: vision, postural control, perception-action, moving room

Small variation of the visual environmental surrounding produces corresponding body sway responses, which was first shown long ago (Lishman \& Lee, 1973). Since this pioneer study, this phenomenon has been observed in infants (Bertenthal \& Bai, 1989), children (Schmuckler, 1997), adults (Lee \& Lishman, 1975), and elderly adults (Prioli, Freitas Júnior, \& Barela, 2005), showing that postural control is coupled to visual information. Schöner (1991) modeled this relationship between visual information and body sway and suggested that the stability of this relationship would provide us with information about how sensory information and body control would be related to each other.

More recently, Schöner, Dijkstra, and Jeka (1998) suggested that movement information captured by the visual system might be interpreted by the postural control system either as self- or object-motion. Self-motion perception occurs as changes in the optical flow are detected and interpreted as resulting from body sway. This information can be used to minimize or to induce body sway based on

\footnotetext{
Barela and Barela are with the Instituto de Ciências da Atividade Física e Esporte, Universidade Cruzeiro do Sul, São Paulo, SP, Brazil. José A. Barela, Rinaldi, and de Toledo are with the Laboratório para Estudos do Movimento, Universidade Estadual Paulista, IB, Departamento de Educação Física, Rio Claro, SP, Brazil.
} 
the projection of the visual scene into the retina (Paulus, Straube, \& Brandt, 1984). On the other hand, object-motion perception occurs as the foreground is interpreted differently from the background surrounding, that is, when individuals interpret that environment motion occurs instead of their body sway (Schöner et al., 1998).

In the self-motion perception mode, the system would respond to the expansion rate of the target or environment in the retina, and it would display a strong and stable coupling between visual information and body sway. Differently, in object-motion mode, in which the environmental surrounding movement is perceived by the individual, the system would couple to this external motion, which does not depend on the postural state, leading to a weak and less stable coupling between visual information and body sway. The suggestion of self- and objectmotion perception was examined in adults (Freitas Júnior \& Barela, 2004), and the results confirmed that the coupling between visual information and body sway is weaker in the object-motion than in the self-motion mode. Similar results were recently observed when participants were asked to resist optical flow influences (Stoffregen, Hove, Schmit, \& Bardy, 2006). Both studies reveal that the coupling strength can be reduced through the intention to resist the sensory stimulus or through knowledge about the moving environment as suggested and mathematically modeled (Schöner et al., 1998).

The finding that postural control is not driven automatically by the sensory stimulus raises questions about the factors that might influence the extent to which postural sway is coupled to the available stimulus. In this case, how would the characteristics of the sensory stimulus influence the effects of intention on the strength coupling between sensory information and body sway? Several studies have experimentally shown that body sway response to sensory stimulus changes as the stimulus amplitude abruptly increases (Oie, Kiemel, Barela, \& Jeka, 2005; Oie, Kiemel, \& Jeka, 2002; Peterka, 2002). Recently, this phenomena has been modeled (Carver, Kiemel, \& Jeka, 2006; Carver, Kiemel, van der Kooij, \& Jeka, 2005), but a complete description of the process underlying such phenomena still needs to be unfolded.

Carver and colleagues (2005) have proposed a model in which the postural system adjusts sensory weights to optimize some observable measure of sway when sensory cue properties change abruptly. This model produced a qualitatively correct response to the moving room with gain dropping and phase remaining roughly constant (around zero at driving frequency of $0.2 \mathrm{~Hz}$ ) with increasing amplitude of the visual stimulus and, therefore, was suggested to capture the dynamics of the sensory reweighting process. A drop in gain with constant phase values was also observed when participants in a moving room environment were told that the room was going to oscillate (Freitas Júnior \& Barela, 2004).

Based on these recent results, it seems that both intention and stimulus characteristics influence the use of sensory information to control body sway. Moreover, in both cases, the postural mechanisms adopted similar strategies, relying less on visual cues, indicating that sensory reweighting may have happened as suggested by the authors in each case (Carver et al., 2006; Carver et al., 2005; Freitas Júnior \& Barela, 2004). Despite these results, the interaction of both 
stimulus characteristics and intention in the use of sensory information to control body sway has not been carefully examined.

Finally, the reweighting process has been examined through abrupt and large increases/decreases of sensory stimulus that could be noticed by the participants (e.g., Oie et al., 2005). In this case, the dynamics of sensory reweighting might have been intentionally influenced by the participant. Differently, in the current study we employed a small stimulus amplitude/velocity that would not provide any awareness to the participants about the visual manipulation. Therefore, the purpose of this study was to examine the influence of both optic flow characteristics and intention on postural control responses. We hypothesized that intention plays a role in reducing coupling strength but is dependent on stimulus characteristics. As the system reweights sensory influences due to abrupt changes in stimulus characteristics (Carver et al., 2006; Oie et al., 2005), we suggest that it also will reweight due to small changes in stimulus characteristics, and in doing so, intention might not be necessary to change the motorsensory coupling in postural control in such situations.

\section{Method}

\section{Participants}

Twenty healthy undergraduate students with no known musculoskeletal injuries or neurological disorders that might have affected their ability to maintain balance participated in the study. All of them had normal or corrected-to-normal vision. Participants were not aware of the study's purpose before participating, and they formed two experimental groups: Low Velocity (LV), in which the participants (19.8 \pm 2.7 years) were exposed to a constant optic flow peak velocity of $0.6 \mathrm{~cm} / \mathrm{s}$, and High Velocity $(\mathrm{HV})$, in which the participants $(19.5 \pm 1.8$ years) were exposed to a constant optic flow peak velocity of $1.0 \mathrm{~cm} / \mathrm{s}$.

\section{Apparatus}

A moving room was used in the study. This room consisted of three walls and a roof mounted on four wheels that slide back and forth on rails, independent of the floor. The dimensions of the room were $2.1 \times 2.1 \times 2.1 \mathrm{~m}$ (height, width, and length). The ceiling was painted white, and the walls were painted black and white in a $20-\mathrm{cm}$-wide vertical stripes pattern. A fluorescent light fixture attached to the center of the room's ceiling illuminated the moving room. A servo-mechanism system (Compumotor) consisting of a controller, a controlled stepper motor, and an electrical cylinder produced the room movement, and this movement was initiated by means of custom software (Compumotor-Motion Architect for Windows).

Body sway and moving room displacement data were obtained through an OPTORAK system (Digital Northern, Inc.) using IRED emitters placed on participants' backs between their scapulae and on the frontal wall of the room. The data were acquired at a frequency of $100 \mathrm{~Hz}$. 


\section{Procedures}

We instructed all participants to maintain an upright position inside of the moving room in their bare feet and with their arms hanging beside their bodies. For both groups, data were acquired for seven repetitions $60 \mathrm{~s}$ apiece. In the first trial, the room was not oscillated, and in the remaining six trials, the room was continuously oscillated backward and forward at a frequency of $0.2 \mathrm{~Hz}$. In the first three trials that the room was oscillated, we instructed the participants to look straight ahead toward a target placed $1 \mathrm{~m}$ away on the front wall at eye level, and they were not aware of the room's oscillation (normal condition). In the remaining three trials that the room was oscillated, we informed the participants about the room oscillation and instructed them to look straight ahead, as in the previous three repetitions, and to avoid oscillating with the room (resist condition).

As mentioned before, the room was oscillated with different constant peak velocities and amplitudes for each of the experimental groups. The constant peak velocity and amplitude were $0.6 \mathrm{~cm} / \mathrm{s}$ and $1.2 \mathrm{~cm}$, respectively, for the LV group and $1.0 \mathrm{~cm} / \mathrm{s}$ and $1.9 \mathrm{~cm}$, respectively, for $\mathrm{HV}$ group.

\section{Data Analysis}

For all seven trials, mean sway amplitude (MSA) was obtained as a measure of overall magnitude of body sway in the anterior-posterior direction. It was calculated by obtaining the standard deviation of body sway signal after the average of body sway position was subtracted from the data points within each trial. In the trials in which the moving room was oscillated, our analyses between the moving room displacement and body sway relationship also focused only on the anteriorposterior direction because this was the direction that the moving room oscillated. To examine the temporal relationship and the influence of visual information on body sway in the normal and resist conditions, we employed four dependent variables: coherence, gain, phase, and stimulus frequency sway amplitude (SFSA). Coherence measured how strongly body sway was coupled to the visual stimulus, calculated at a frequency of $0.2 \mathrm{~Hz}$. Coherence values close to 1 indicate that the signals demonstrate a strong dependency between them. Conversely, values close to zero indicate that the signals demonstrate a weak or no dependency between them. Gain measured how much the moving room oscillation influenced body sway and was calculated as a ratio between body sway and moving room amplitude spectrum at a frequency of $0.2 \mathrm{~Hz}$. Gain values smaller or larger than 1 indicate that body sway spectrum amplitude was smaller or larger than the visual stimulus spectrum, respectively. Phase provided information about the temporal relationship between body sway and moving room oscillation. Phase values larger or smaller than zero indicate that body sway was ahead or behind the moving room oscillation, respectively. Phase values equal to zero indicate no time lag between body sway and moving room oscillation. SFSA indicated the spectral magnitude of body sway at the driving frequency, that is, at $0.2 \mathrm{~Hz}$. All the dependent variables were calculated using custom software written in Matlab (Math Works, Inc., version 7.0).

A Fisher transformation was employed on the dependent variable coherence to normalize the data distribution. One MANOVA and two ANOVAs were 
employed, having group (LV and HV) and condition (normal and resist) as factors, with the last factor treated as a repeated measure. The MANOVA dependent variables were coherence and gain, and the ANOVAs dependent variables were phase and SFSA. Another ANOVA was employed, having group (LV and HV) and condition (no movement of the room, normal, and resist) as factors, also with the last factor treated as a repeated measure, and the dependent variable was MSA. When applicable, honestly significant difference Tukey post hoc tests were employed. An alpha level of .05 was used for all statistical tests, which were performed using the SPSS software (SPSS 10.0 for Windows).

\section{Results}

The moving room oscillation induced body sway in participants in both groups and in normal and resist conditions. In the normal trials, none of the participants reported any awareness about the movement of the room. Figure 1 presents sample time series and amplitude spectra of the moving room oscillation and body sway for one participant of LV (Figure $1 \mathrm{~A}-\mathrm{D}$ ) and HV groups (Figure $1 \mathrm{E}-\mathrm{H}$ ) in both normal (Figure 1 A, B, E, F) and resist conditions (Figure $1 \mathrm{C}, \mathrm{D}, \mathrm{G}, \mathrm{H}$ ). The overlaid time series for both participants show that body sway coupled to the moving room oscillation in both conditions (Figure $1 \mathrm{~A}, \mathrm{C}, \mathrm{E}, \mathrm{G}$ ). In addition, spectral plots for both participants in the normal condition show a defined peak in the body sway signal at the moving room oscillation frequency of $0.2 \mathrm{~Hz}$, whereas in the resist condition, both participants presented a broader band of frequencies (Figure $1 \mathrm{D}, \mathrm{H}$ ) than in the normal condition (Figure $1 \mathrm{~B}, \mathrm{~F}$ ).

Figure 2 presents mean $( \pm S D)$ coherence and gain values, respectively, for LV and $\mathrm{HV}$ groups in both normal and resist conditions. The MANOVA revealed a significant group effect, Wilks's lambda $=0.57, F(2,17)=6.55, p=.008$; condition effect, Wilks's lambda $=0.55, F(2,17)=7.06, p=.006$; and group and condition interaction, Wilks's lambda $=0.57, F(2,17)=6.30, p=.009$. Univariate analyses indicated group effect for coherence, $F(1,18)=4.71, p=.044$, and gain, $F(1,18)=13.72, p=.002$. Univariate analyses indicated a condition effect only for gain, $F(1,18)=13.99, p=.002$. Finally, univariate analyses indicated a group and condition interaction only for gain, $F(1,18)=7.61, p=.013$. Coherence values were significantly higher for the LV group compared with the HV group (Figure 2A). In addition, post hoc tests indicate that LV group gain values were significantly lower in the resist condition than in the normal condition and that HV group gain values were not different between both conditions. Finally, LV group gain values were significantly higher than $\mathrm{HV}$ group values in both normal and resist conditions (Figure $2 \mathrm{~B}$ ).

Figure 3 depicts mean $( \pm S D)$ phase values for LV and HV groups in both normal and resist conditions. ANOVA revealed a significant condition effect, $F(1$, $18)=5.46, p=.03$, but no significant group effect, $F(1,18)=2.93, p=.10$, or condition and group interaction, $F(1,18)=0.02, p=.90$. In the normal condition, body sway was in-phase or slightly behind the moving room oscillation, and in the resist condition, body sway was slightly ahead of the moving room oscillation.

Figure 4 depicts mean $( \pm S D)$ SFSA values for both groups in both conditions. ANOVA revealed a significant condition effect, $F(1,18)=13.15, p=.002$, no 

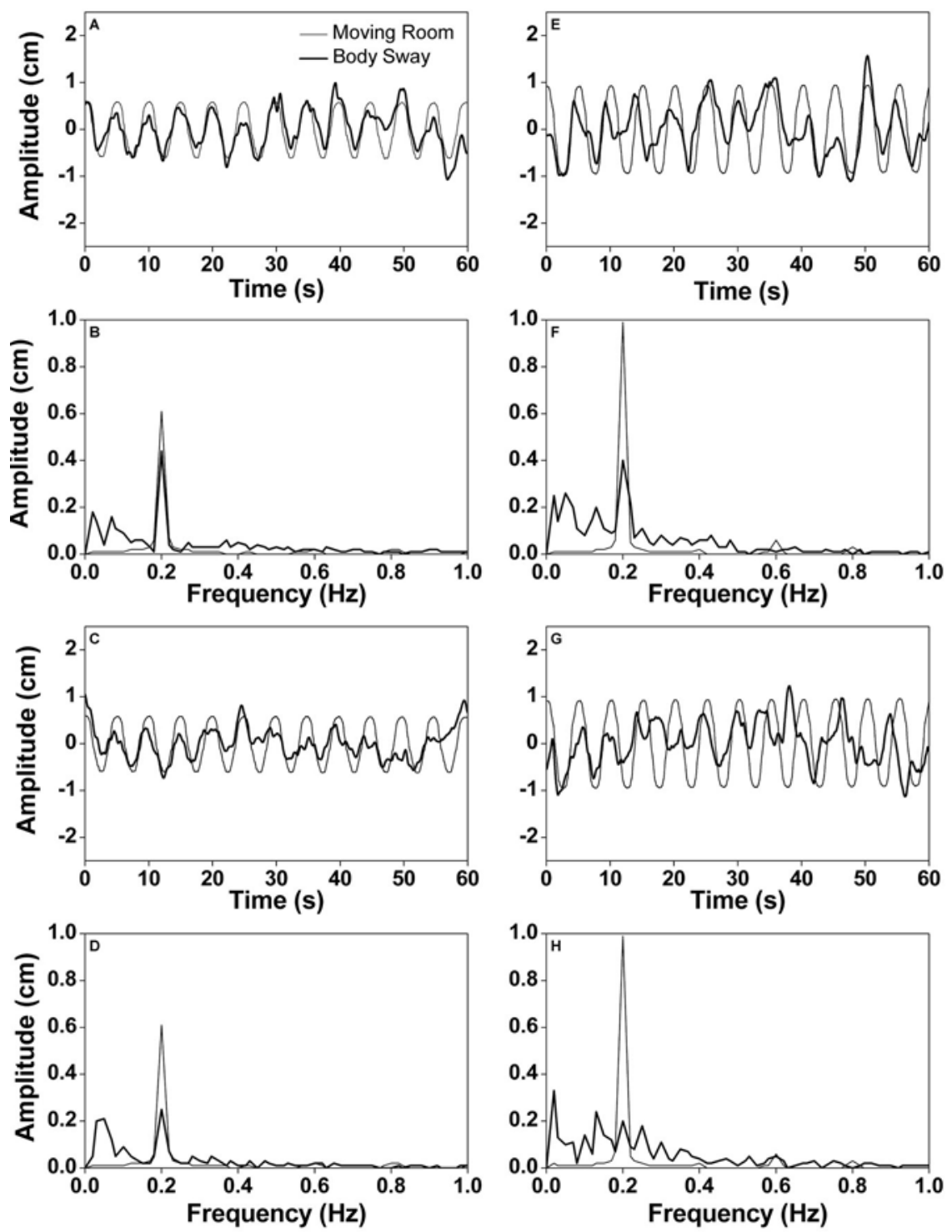

Figure 1 - Sample time series and amplitude spectra of moving room and body sway in the anterior-posterior direction for both Low Velocity (A-D) and High Velocity (E-H) groups in normal (A, B, E, and F) and resist $(\mathrm{C}, \mathrm{D}, \mathrm{G}$, and $\mathrm{H})$ conditions. 

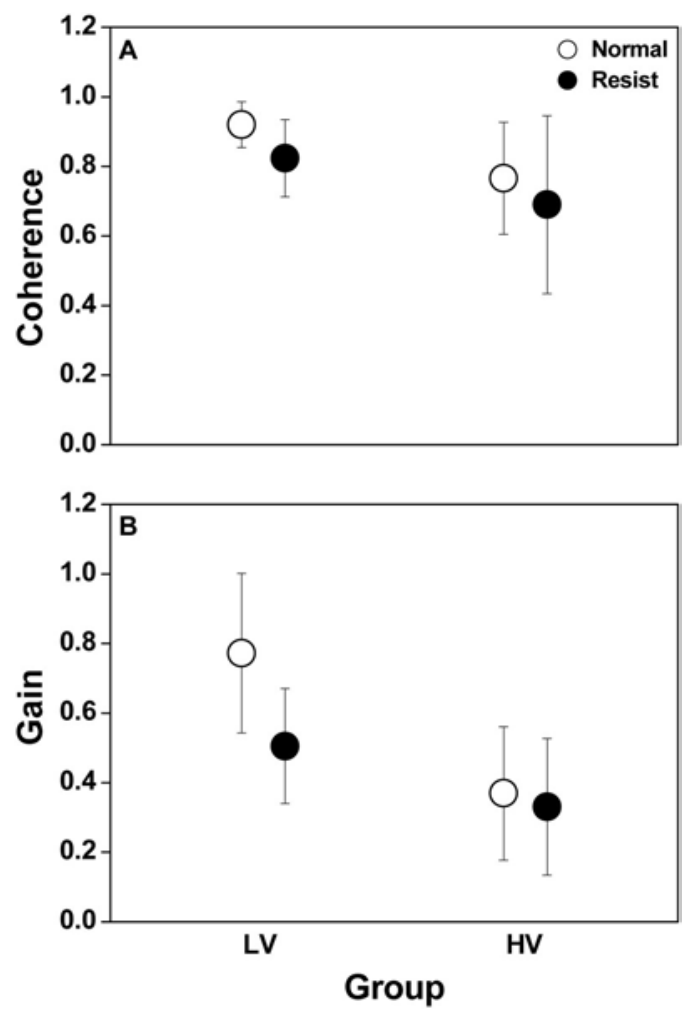

Figure 2 - Mean $( \pm S D)$ coherence and gain values between the moving room oscillation and body sway in the anterior-posterior direction for both Low Velocity (LV) and High Velocity (HV) groups in normal and resist conditions.

significant group effect, $F(1,18)=0.14, p=.71$, and condition and group interaction tendency, $F(1,18)=3.59, p=.07$. The spectral magnitude of body sway was higher in the normal condition than in the resist condition.

Finally, Figure 5 depicts mean $( \pm S D)$ MSA values for LV and HV groups in the no room movement, normal, and resist conditions. ANOVA revealed a significant condition effect, $F(2,36)=26.49, p<.001$, but no significant group effect, $F(1,18)=0.60, p=.44$, and condition and group interaction, $F(2,36)=1.78, p=$ .18. Post hoc tests indicated that although there was no difference between normal and resist conditions, body sway in these two conditions was larger than in the no room movement condition.

\section{Discussion}

The goal of this study was to examine the influence of both optic flow characteristics and intention on postural control responses. Our results showed that intention influences the coupling between visual information and body sway, but this 


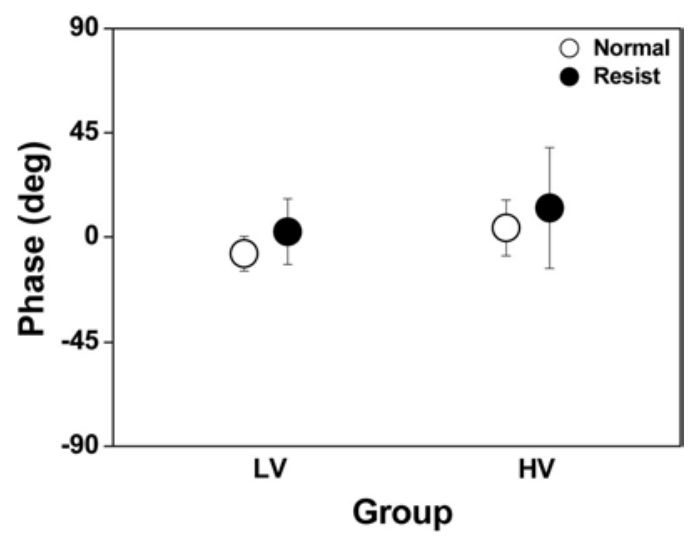

Figure 3 - Mean $( \pm S D)$ phase values between the moving room oscillation and body sway in the anterior-posterior direction for both Low Velocity (LV) and High Velocity (HV) groups in normal and resist conditions.

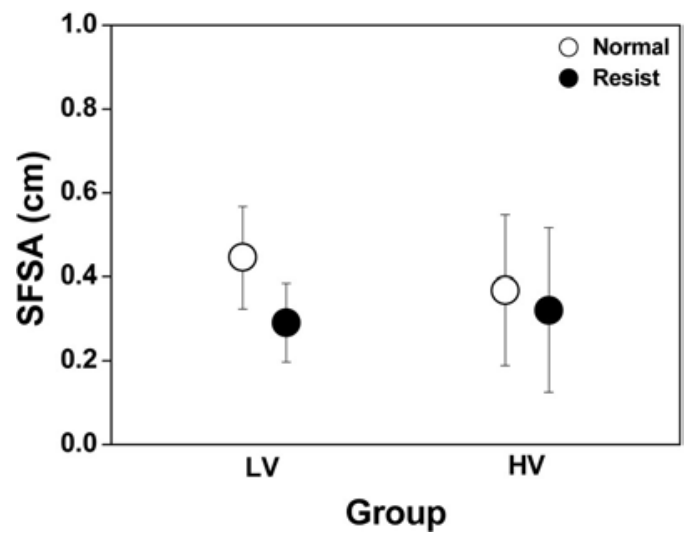

Figure 4 - Mean $( \pm S D)$ stimulus frequency sway amplitude (SFSA) values of the body sway in the anterior-posterior direction for both Low Velocity (LV) and High Velocity (HV) groups in normal and resist conditions.

influence depends on the characteristics of the stimulus. Specifically, intention only played a role in affecting the magnitude of body sway at the driving frequency in the visual low velocity stimulus. When velocity of the moving room was slightly higher, the postural control system had already captured this information and reduced the coupling strength and the stimulus influences even before any request for the participants to avoid the visual stimulus.

Manipulation of visual information provided by the moving room induced body sway in all participants, and body sway was related and coherent to the visual information. This finding is in agreement with several other studies (e.g., Dijkstra, Schöner, \& Gielen, 1994; Freitas Júnior \& Barela, 2004; Lishman \& Lee, 1973; Stoffregen, 1985). However, we also observed that the magnitude 


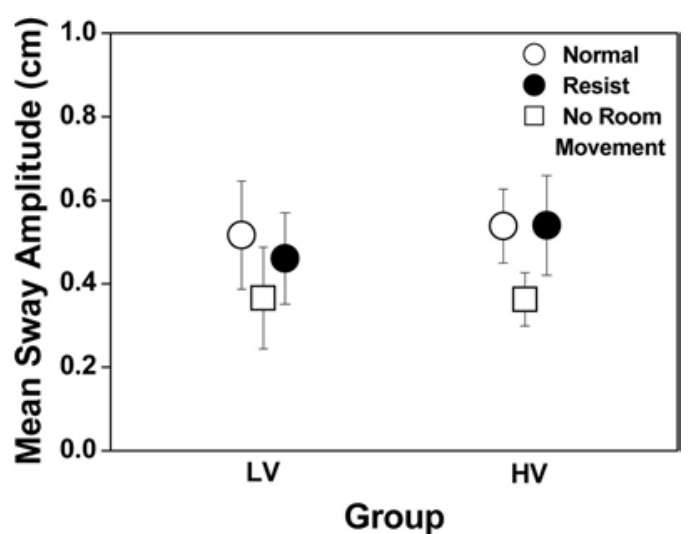

Figure 5 - Mean $( \pm S D)$ mean amplitude sway (MAS) values of the body sway in the anterior-posterior direction for both Low Velocity (LV) and High Velocity (HV) groups in the no room movement, normal, and resist conditions.

influences of imposed optic flow on body sway depend on the characteristics of the visual stimulus. Our results in the normal condition clearly showed that the influence of the visual stimulus on body sway (gain and a tendency of SFSA values) was significantly lower when the room was oscillated at $1 \mathrm{~cm} / \mathrm{s}$ than when it was oscillated at $0.6 \mathrm{~cm} / \mathrm{s}$. In this case, the postural control system, based on the characteristics of high velocity of the visual stimulus, decreased the influence of this stimulation on body sway. Moreover, the coupling strength between the visual stimulus and body sway (coherence) also decreased when the room was oscillated with high velocity compared with the low velocity condition.

Changes in coupling strength and influences of sensory stimulus on body sway have been suggested and modeled (Schöner et al., 1998) and observed either when participants were informed about the room's movement (Freitas Júnior \& Barela, 2004) or requested to resist its influence (Stoffregen et al., 2006). In these cases, intention was showed to play an important role in reweighting the influence of a specific sensory stimulus such as changing from the self- to object-motion mode of postural control functioning (Carver et al., 2006; Schöner et al., 1998). On the other hand, despite being considered critical for flexible postural control (Horak, Diener, \& Nashner, 1989), only recently has sensory reweighting due to the stimulus characteristics been properly discussed and implemented to postural control functioning (Carver et al., 2006; Oie et al., 2005). In this way, our results indicate that the postural control system also reweights the sensory stimulus influences based on the stimulus characteristics even at small amplitude/velocity manipulation of the stimulus. Moreover, our results suggest that such dynamics of sensory reweighting do not involve intention because none of the participants were aware or able to verbalize any manipulation or change in visual surrounding before being told about the room movement.

More interestingly, however, it is that intention or willingness may not play a role in reducing the stimulus influences on postural sway if the postural control system has already changed the weight of a sensory stimulus based on its 
characteristics. In the current study, the influence of the visual information was downweighted when the stimulus velocity was high, and the request to resist the room oscillation did not change the influence and the coupling strength between the visual stimulus and body sway. Such a mechanism is quite clever because it provides the system ways to prevent sensory influences without a direct intervention of high centers related to postural control. Body sway is still coherent and influenced by the sensory stimulus but not linearly to the same magnitude of sensory stimulus increasing. Only recently has the functioning of such mechanisms been examined more carefully in large stimulus changes (Carver et al., 2006; Oie et al., 2005), and we can add that such nonlinearity of response to visual manipulation can also be observed in conditions of small stimulus amplitude.

Even though the postural control system might use both intention and stimulus characteristics to change a sensory stimulus influence on body sway, it still seems not to be able to fully ignore or avoid influences from it. The influence of visual stimulus on sway changed due to request and stimulus characteristics, but still, corresponding body oscillation to the stimulus was observed as shown by high coherence values. Moreover, the overall body sway was similar in both normal and resist conditions but still larger than when there was no driving visual information. These findings indicate that despite being less influenced by the driving signal, the postural control cannot totally avoid sensory influences and produces body sway in frequencies other than the one specified by the moving room. Actually, the decrease in gain and coherence indicates that intention and stimulus characteristics seem to change this influence just a bit. Yet, it seems that body sway was reduced at the driving frequency, as shown by the SFSA values, but still is manifested in other frequencies because the overall body sway did not change between conditions in which the room was moved and it was larger than when no signal was manipulated. Therefore, the postural control system pays a price in avoiding visual manipulation influences, even for a small magnitude, in such a way that body sway is still manifested in frequencies other than the driving stimulus frequency.

Finally, besides not being able to completely avoid visual influence on body sway, intention changes the temporal relationship between the room oscillation and body sway. In this case, phase values revealed that in the normal condition, body sway and the moving room were oscillating together without any delay, whereas in the resist condition, body sway was slightly ahead of the moving room. These results were different from previous studies (Freitas Júnior \& Barela, 2004; Stoffregen et al., 2006) and indicate that when one is aware or asked to resist visual influence, he or she seems to try to anticipate the room oscillation and, in doing so, moves ahead compared with when he or she was not aware or asked to resist the visual stimulus.

In short, our results showed that both intention and stimulus characteristics may affect the use of sensory stimulus by the postural control system. However, the influence of intention is dependent on the characteristics of the stimulus that the postural control system has been exposed to because once the postural control system has been influenced by some of the stimulus characteristics, intention does not change the influence of sensory information on body sway. 


\section{References}

Bertenthal, B.I., \& Bai, D.L. (1989). Infant's sensitivity to optical flow for controlling posture. Developmental Psychology, 25(6), 936-945.

Carver, S., Kiemel, T., \& Jeka, J.J. (2006). Modeling the dynamics of sensory reweighting. Biological Cybernetics, 95(2), 123-134.

Carver, S., Kiemel, T., van der Kooij, H., \& Jeka, J.J. (2005). Comparing internal models of the dynamics of the visual environment. Biological Cybernetics, 92, 147-163.

Dijkstra, T.M., Schöner, G., \& Gielen, C.C. (1994). Temporal stability of the actionperception cycle for postural control in a moving visual environment. Experimental Brain Research, 97, 477-486.

Freitas Júnior, P.B., \& Barela, J.A. (2004). Postural control as a function of self- and objectmotion perception. Neuroscience Letters, 369, 64-68.

Horak, F.B., Diener, H.C., \& Nashner, L.M. (1989). Influence of central set on human postural responses. Journal of Neurophysiology, 62(4), 841-853.

Lee, D.N., \& Lishman, J.R. (1975). Visual proprioceptive control of stance. Journal of Human Movement Studies, 1, 87-95.

Lishman, J.R., \& Lee, D.N. (1973). The autonomy of visual kinaesthesis. Perception, 2, 287-294.

Oie, K.S., Kiemel, T., Barela, J.A., \& Jeka, J.J. (2005). The dynamics of sensory reweighting: A temporal asymmetry. Gait \& Posture, 21(1), S29.

Oie, K.S., Kiemel, T., \& Jeka, J.J. (2002). Multisensory fusion: Simultaneous re-weighting of vision and touch for the control of human posture. Cognitive Brain Research, 14, 164-176.

Paulus, W.M., Straube, A., \& Brandt, T. (1984). Visual stabilization of posture: Physiological stimulus characteristics and clinical aspects. Brain, 107, 1143-1163.

Peterka, R.J. (2002). Sensorimotor integration in human postural control. Journal of Neurophysiology, 88, 1097-1118.

Prioli, A.C., Freitas Júnior, P.B., \& Barela, J.A. (2005). Physical activity and postural control in the elderly: Coupling between visual information and body sway. Gerontology, $51,145-148$.

Schmuckler, M.A. (1997). Children's postural sway in response to low- and high-frequency visual information for oscillation. Journal of Experimental Psychology. Human Perception and Performance, 23(2), 528-545.

Schöner, G. (1991). Dynamics theory of action-perception patterns: The "moving room" paradigm. Biological Cybernetics, 64, 455-462.

Schöner, G., Dijkstra, T.M.H., \& Jeka, J.J. (1998). Action-perception patterns emerge from coupling and adaptation. Ecological Psychology, 10(3-4), 323-346.

Stoffregen, T.A. (1985). Flow structure versus retinal location in the optical control of stance. Journal of Experimental Psychology. Human Perception and Performance, 11(5), 554-565.

Stoffregen, T.A., Hove, P., Schmit, J., \& Bardy, B.G. (2006). Voluntary and involuntary postural responses to imposed optic flow. Motor Control, 10, 24-33. 
Copyright of Motor Control is the property of Human Kinetics Publishers, Inc. and its content may not be copied or emailed to multiple sites or posted to a listserv without the copyright holder's express written permission. However, users may print, download, or email articles for individual use. 